\title{
Compósitos de polipropileno e fibras curtas de sílica
}

\section{Polypropylene and silica short fibers composites}

\author{
Mariana Gava Segatelli ${ }^{1}$; Cristiane Aparecida da Silva ${ }^{2}$; Inez Valéria Pagotto \\ Yoshida $^{3}$; Maria do Carmo Gonçalves ${ }^{4}$
}

\begin{abstract}
Resumo
As propriedades mecânicas dos compósitos dependem fortemente da natureza da interface carga-matriz polimérica. Uma interface que apresenta forte ligação entre a carga e a matriz geralmente contribui para melhores propriedades mecânicas dos compósitos. Neste trabalho foi utilizado o polipropileno, PP, como matriz polimérica e como carga empregou-se fibras curtas de sílica, FS, que é um produto constituído de sílica biogênica amorfa. Com a finalidade de promover melhor adesão interfacial carga-matriz, os compósitos preparados com FS foram comparados com os similares obtidos com fibras previamente modificadas com viniltrimetoxissilano. Todos os compósitos foram preparados por processos de extrusão e moldagem por injeção, sendo suas propriedades mecânicas avaliadas por ensaios mecânicos de tração, segundo a norma ASTM D 268, utilizando-se um planejamento fatorial fracionário $2^{5-1}$. Os fatores estudados foram composição da fibra na matriz, modificação superficial da fibra, degasagem, fluxo de injeção e velocidade de ensaio de tração. A superfície de fratura criogênica dos compósitos foi investigada por microscopia eletrônica de varredura com fonte de emissão de campo, FESEM. A modificação das fibras promoveu uma melhor adesão interfacial fibra-matriz, o que resultou em propriedades mecânicas otimizadas em relação aos compósitos similares obtidos com fibras naturais, indicando ser este o fator mais importante para o melhor desempenho mecânico dos compósitos.

Palavras-chave: Fibras curtas de sílica. Polipropileno. Planejamento fatorial fracionário.
\end{abstract}

\begin{abstract}
The mechanical properties of composites strongly depend on the type of filler-polymeric matrix interface. An interface characterized by a strong bond between the filler and the matrix generally results in better mechanical properties. In this work, polypropylene, PP, was used as polymeric matrix and silica short fibers, SF, which are amorphous biogenic silica, were employed as filler. In order to promote a better filler-matrix interfacial adhesion, these fibers were previously modified with vinyltrimethoxysilane. The composites were prepared by extrusion and injection molding processes and their mechanical properties were evaluated according to tensile testing, ASTM D 268, by means a fractional factorial design $\left(2^{5-1}\right)$. The studied factors included fiber content in the matrix, fiber surface modification, injection flow and testing rate. The composite cryogenic fractured surfaces were investigated by field emission scanning electron microscopy, FESEM. The fiber modification promoted a better fiber-matrix interfacial adhesion, resulting in optimized mechanical properties in relation to the others. In this way, the fiber modification was the most important factor on the mechanical performance of the composites.
\end{abstract}

Keywords: Silica short fibers. Polypropylene. Fractional factorial design.

\footnotetext{
${ }^{1}$ Professora da Universidade Estadual de Londrina; mariana@uel.br

${ }^{2}$ Pós-Doutoranda do Instituto de Física da Universidade de São Paulo, Campus São Carlos

${ }^{3}$ Professora do Instituto de Química da Universidade Estadual de Campinas

${ }^{4}$ Professora do Instituto de Química da Universidade Estadual de Campinas
} 


\section{Introdução}

A utilização de cargas em matrizes termoplásticas, como em matrizes de polipropileno, tem sido um método eficiente para obtenção de materiais com melhores propriedades mecânicas e também para a redução de custos dos mesmos (HUFENBACH et al., 2011). As cargas mais usuais em polímeros termoplásticos incluem talco, carbonato de cálcio, mica, wollastonita, argilas, sílicas, fibras curtas e longas de vidro, entre outras (WANG et al., 2011b; UNAL; MIMAROGLU; ALKAN, 2004). O uso de fibras de vidro em termoplásticos de engenharia tem reconhecida aplicabilidade nas indústrias automotiva e elétrica, sendo também uma das cargas mais exploradas em estudos acadêmicos (WANG et al., 2011b; ARBELAIZ et al., 2005; THOMASON, 2005; OTA; AMICO; SATYANARAYANA, 2005; ROHDE, et al., 2011; ZONG; YANG; DAI, 2011).

As propriedades mecânicas finais dos compósitos poliméricos dependem basicamente de parâmetros como natureza das cargas, distribuição e orientação das cargas na matriz polimérica e adesão interfacial carga-matriz. Melhores propriedades mecânicas são obtidas pela transferência de tensões entre matriz e carga. Para que haja eficiência nesta transferência é necessário que haja uma boa adesão na interface polímerocarga, uma vez que essa transferência ocorre na região de contato entre as fases (CHAWLA, 1987; SEVER et al., 2012). Como as cargas geralmente apresentam caráter hidrofílico e os polímeros, em sua maioria, hidrofóbicos, torna-se difícil obter adesão interfacial adequada entre esses componentes, comprometendo conseqüentemente, as propriedades mecânicas dos compósitos (UNAL; MIMAROGLU, 2004). No caso específico de fibras de vidro dispersas em matriz hidrofóbica de polipropileno, PP, não é obtida interação interfacial satisfatória, uma vez que essas fibras apresentam grupos polares silanóis $(\mathrm{SiOH})$ em sua superfície.

Uma rota muito comum e eficiente para aumentar a adesão entre carga e matriz polimérica é pela modificação química da superfície da carga. Uma classe de agentes modificadores muito empregados em superfícies polares hidroxiladas é constituída pelos organossilanos. Neste caso, os organossilanos atuam como agentes de acoplamento entre carga e matriz (PLUEDDEMANN, 1991; WANG et al., 2011a).

Organossilanos de fórmula geral $\left.\mathrm{Y}_{\left(\mathrm{CH}_{2}\right.}\right)_{3} \mathrm{SiX}_{3}$, é uma classe de agentes de acoplamento onde $\mathrm{X}$ representa os grupos hidrolisáveis como alcóxidos ou cloretos, importantes para reagir com a superfície hidroxilada de cargas, e Y corresponde a um grupo orgânico funcional de interesse, com reatividade adequada para interagir com a matriz polimérica, como por exemplo: amina, epóxido, vinil, etc. Organotrialcoxissilanos, com as mais diferentes funções $\mathrm{Y}$, têm sido muito empregados em modificações de superfície (SENER et al., 2005) e como agentes de acoplamento (BALAS et al., 2006; BOUZA et al., 2008). Usualmente são empregados como solução de oligômeros (prépolímeros) (REDONDO et al., 2002), ricos em silanóis $(\mathrm{Si}-\mathrm{OH})$ e/ou alcoxissilanos ( $\mathrm{Si}-\mathrm{OR})$. Os grupos $\mathrm{Si}-\mathrm{OH}$ e/ou Si-OR do pré-polímero podem sofrer condensação entre si e com grupos silanóis presentes na superfície de cargas hidroxiladas, como por exemplo, fibras de vidro, formando assim ligações siloxano (SiOSi) na interface. Deste modo, o agente de acoplamento modifica as fibras de vidro inserindo grupos orgânicos contendo a funcionalidade desejada na superfície permitindo que ocorra interação mais efetiva entre a matriz e a carga. Devido a essa dupla funcionalidade, os organossilanos podem atuar como ponte entre carga e matriz. A Figura 1 ilustra um esquema geral da modificação da superfície de fibras de vidro, utilizando organotrialcoxissilano como agente de acoplamento.

Neste trabalho, foi utilizado polipropileno isotático (PP) como matriz termoplástica e como carga foi empregada fibra curta de sílica, FS, que é um produto constituído de sílica amorfa 
biogenicamente formada, denominada espongilito. Esta fibra possui características morfológicas semelhantes às fibras curtas de vidro, podendo ser utilizada como uma carga alternativa de reforço em termoplásticos. Com a finalidade de ajustar as condições de processamento das fibras com a matriz polimérica, para avaliar o desempenho mecânico dos compósitos, empregou-se um

Figura 1 - Reação esquemática entre trialcoxissilano e fibra de vidro.

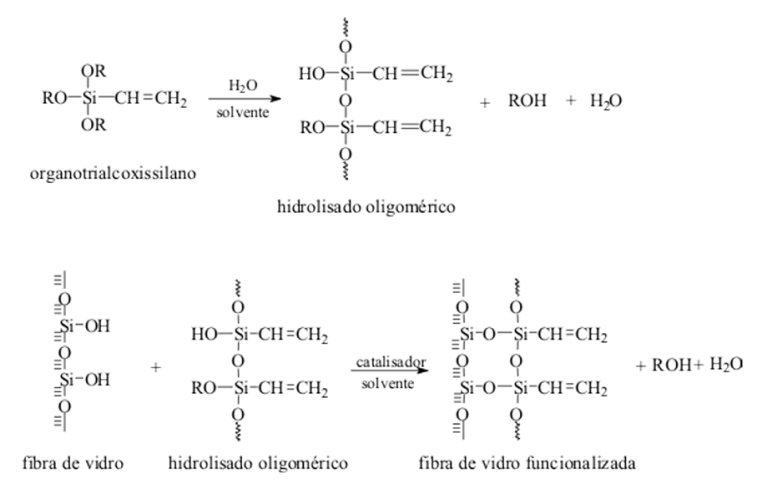

Fonte: Dados do autor.

\section{Experimental}

\section{Materiais de partida}

Polipropileno isotático, PP, utilizado como matriz polimérica termoplástica, $(\mathrm{H} 301, \rho=0,91$ $\mathrm{g} / \mathrm{cm}^{3}$ e IF $=10 \mathrm{~g} / 10 \mathrm{~min}$ ), foi de procedência Braskem (São Paulo, SP, Brasil). As fibras curtas de sílica, FS, foram adquiridas da empresa Cerâmica São Caetano, coligada à Magnesita S.A (Contagem, MG, Brasil). Essas fibras são constituídas de sílica amorfa e apresentam-se como agulhas cilíndricas, tubulares, com ambas as extremidades afinadas. Suas dimensões médias para comprimento e diâmetro são de $150 \mu \mathrm{m}$ e $12 \mu \mathrm{m}$, respectivamente. Para a modificação superficial das fibras foram empregados o agente de acoplamento vinitrimetoxissilano, VTS, de procedência Dow Corning do Brasil (Hortolândia, SP, Brasil) e o catalisador tetran-butóxido de titânio, TBT, de procedência Du Pont (Wilmington, DE, USA). Como solvente foi utilizado tetrahidrofurano, THF, fornecido pela Carlo Erba (Rodano, MI, Itália).

\section{Modificação das fibras}

Inicialmente, realizou-se a hidrólise parcial do VTS, em THF, adicionando-se água na relação molarVTS : $\mathrm{H}_{2} \mathrm{O}$ de $1: 1$, em um balão contendo uma solução de $\sim 0,62 \%$ (em massa) de VTS em THF. Manteve-se esta mistura sob agitação magnética, em atmosfera de argônio, por $3 \mathrm{~h}$, à temperatura ambiente. Em seguida, a FS, previamente seca a $120^{\circ} \mathrm{C}$ por $48 \mathrm{~h}$, foi adicionada ao balão reacional, juntamente com $2 \%$ (em massa) de catalisador TBT. Utilizou-se $0,5 \%$ (em massa) de VTS em relação à FS. A reação de modificação permaneceu sob agitação mecânica, por $24 \mathrm{~h}$, à temperatura ambiente. Após este período, foi efetuada a filtração da fibra, sendo em seguida submetida à extração de possível fração de silano não ancorada covalentemente à superfície da fibra. A extração foi feita com THF, sob agitação mecânica, por três vezes. Em seguida, a fibra foi separada por filtração e seca por $24 \mathrm{~h}$, à temperatura ambiente, para evaporação do solvente. Finalmente, a fibra foi seca em estufa a $60^{\circ} \mathrm{C}$, por $\sim 48 \mathrm{~h}$.

\section{Preparação dos compósitos}

Os compósitos, $\mathrm{PP} / \mathrm{FS}$, bem como o polímero puro, PP, foram processados em uma extrusora de rosca simples Wortex $(\mathrm{L} / \mathrm{D}=32,30 \mathrm{~mm}$ de diâmetro) com degasagem e 5 zonas de aquecimento $\left(200,210,220,230\right.$ e $240^{\circ} \mathrm{C}$, zonas 1 a 5 , respectivamente). Após o processamento, o material foi granulado e moldado por injeção utilizando uma injetora Arburg Allrounder M-250 com 5 zonas de aquecimento $(215,220,220,230 \mathrm{e}$ $220^{\circ} \mathrm{C}$ ). O material foi injetado em corpos de prova em formato apropriado para ensaios mecânicos de 
tração, segundo as especificações da norma ASTM D-638. Após a moldagem por injeção, os corpos de prova foram acondicionados por $48 \mathrm{~h}$ em sala climatizada a $(23 \pm 2)^{\circ} \mathrm{C}$ e $(50 \pm 5) \%$ de umidade relativa. Após este período, os ensaios mecânicos de tração foram realizados em uma máquina universal de ensaios EMIC-DL2000, analisandose parâmetros usuais como módulo de elasticidade, tensão na força máxima, alongamento na ruptura e tensão na ruptura.

\section{Análise do desempenho mecânico dos compósitos}

Para analisar os resultados dos ensaios mecânicos de tração, ou seja, o desempenho mecânico dos compósitos foi aplicado um planejamento fatorial fracionário $2^{5-1}$ (METIN et al., 2004). Neste planejamento foram estudados diversos fatores, cada um deles em dois níveis. Os fatores analisados foram composição da fibra na matriz polimérica (15 e 30\% em massa), modificação superficial da fibra (modificada e não modificada), degasagem (aberta e fechada), fluxo de injeção (3 e $10 \mathrm{~cm}^{3} / \mathrm{s}$ ) e velocidade de ensaio de tração $(10$ e 50 mm/s).

\section{Análise morfológica}

Para analisar a morfologia dos materiais, os compósitos PP/FS contendo fibras não modificadas e modificadas com VTS foram fraturados em nitrogênio líquido e suas superfícies de fratura foram recobertas com uma fina camada de carbono e da liga ouro/paládio, utilizando um metalizador Bal-Tec MED 020. A análise das fraturas criogênicas dos compósitos foi realizada por microscopia eletrônica de varredura com fonte de emissão de campo, (FESEM), utilizando um microscópio Jeol JSM-6340F operando com voltagem de aceleração de $3 \mathrm{kV}$.

\section{Resultados e Discussão}

Para analisar o efeito da introdução de fibras de sílica na matriz polimérica de PP e das condições de processamento nas propriedades mecânicas dos compósitos, foi escolhido um planejamento fatorial 25-1 com relação geradora $I=12345$, cujos resultados estão compilados na Tabela 1 . Utilizando-se estes resultados e com o emprego do programa STATISTICA 6.0 foram avaliados os efeitos dos fatores estudados nas propriedades mecânicas do material.

A Figura 2 apresenta os valores correspondentes à distribuição normal padronizada $(Z)$ em função dos valores calculados dos efeitos relacionados ao alongamento na ruptura e módulo de elasticidade. A escolha deste gráfico é justificada pelo fato dos experimentos terem sido realizados sem repetições (BARROS NETO; SCARMINIO; BRUNS, 2002). 
Tabela 1 - Resultados dos ensaios de tração dos compósitos PP/FS em função dos seguintes fatores: composição da fibra, modificação superficial da fibra, degasagem, fluxo de injeção e velocidade de ensaio.

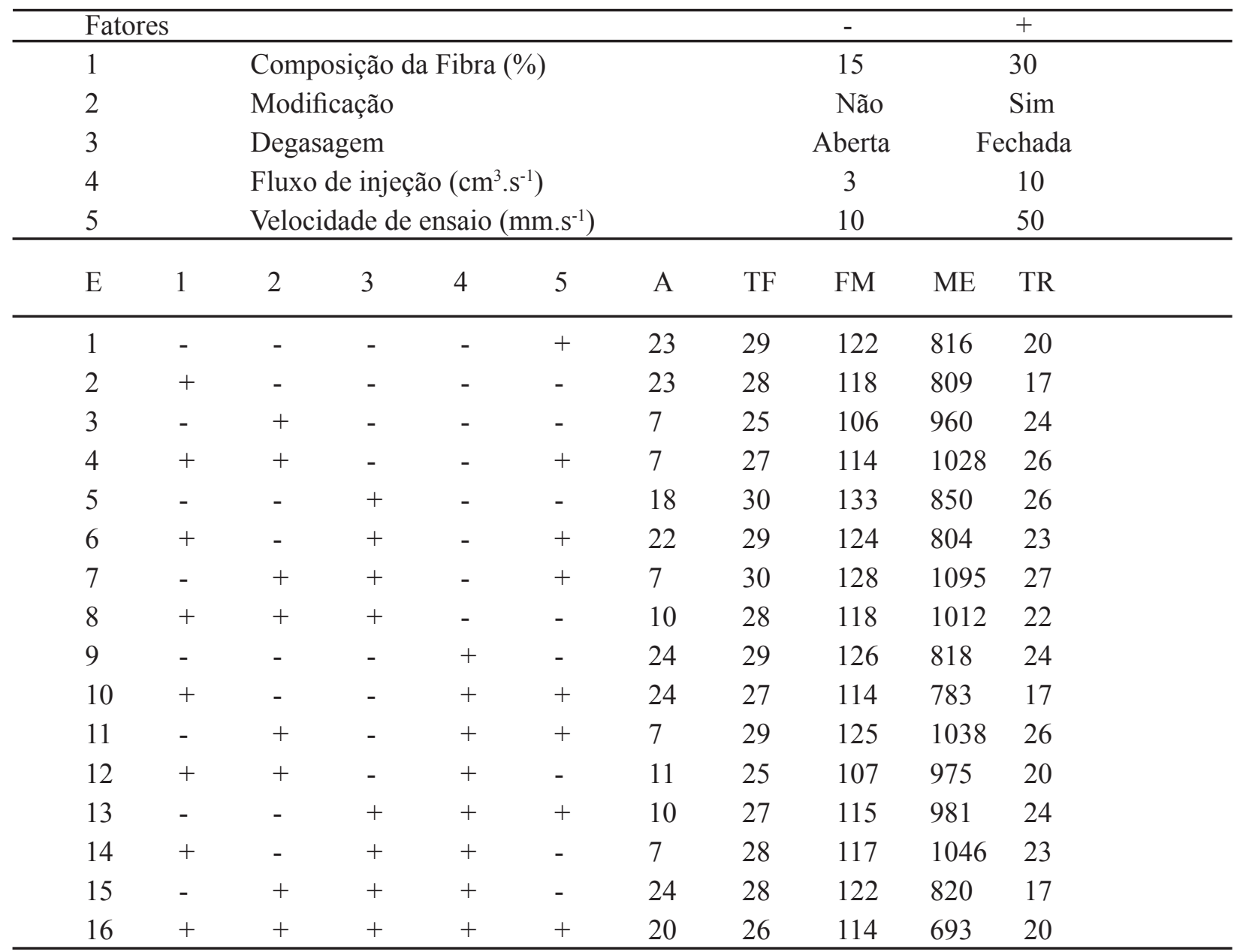

$\mathrm{E}=$ ensaios, $\mathrm{A}(\%)=$ alongamento, $\mathrm{TF}(\mathrm{MPa})=$ tensão na força máxima, $\mathrm{FM}(\mathrm{kgf})=$ força máxima, $\mathrm{ME}(\mathrm{MPa})=$ módulo de elasticidade, $\mathrm{TR}(\mathrm{MPa})=$ tensão na ruptura.

Fonte: Dados do autor.

Figura 2 - Gráfico normal dos efeitos para alongamento na ruptura (a) e módulo de elasticidade (b).
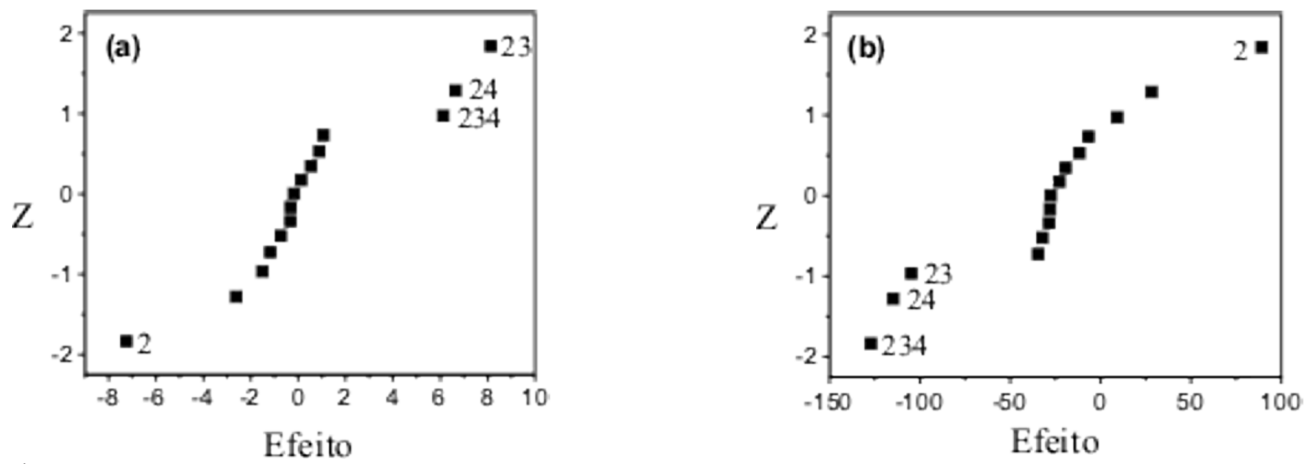

Fonte: Dados do autor.

Efeito 
Nota-se que, em ambos os casos, os pontos centrais correspondentes a outros efeitos estão agrupados ao redor do ponto zero, o que representa que as combinações destes últimos têm pouca influência nos parâmetros estudados. Pode-se considerar que a origem destes pontos é uma população normal de média zero. Em outras palavras, eles representam efeitos sem significado físico. Já os pontos não agrupados, referentes à modificação da fibra (2) e os efeitos de interação envolvidos com esse fator (23, 24 e 234), são considerados significativos, apresentando assim influência nas propriedades mecânicas dos compósitos. Para a análise desses resultados foram avaliados apenas o alongamento na ruptura e o módulo de elasticidade, uma vez que os demais parâmetros não apresentaram diferenças significativas entre as respectivas medidas. Tendo em vista que a composição da fibra estudada não revelou ser um fator importante nas propriedades mecânicas, pode-se considerar como um resultado positivo, já que materiais com boas propriedades mecânicas podem ser obtidos com a menor proporção de fibra, o que contribui para a redução de custos dos experimentos.

Os valores mínimos e máximos de módulo de elasticidade referentes aos compósitos contendo fibras não modificadas e fibras modificadas com VTS foram 783 e $1095 \mathrm{MPa}$, respectivamente. Além disso, foi verificado um aumento no valor deste parâmetro em comparação ao PP puro, 687 MPa. O módulo de elasticidade dos compósitos é uma propriedade mecânica que está relacionada à rigidez do material. Neste caso, o aumento no valor do módulo foi conseqüência de introdução da carga rígida na matriz polimérica de PP, uma vez que as cargas apresentam módulo maior em relação aos polímeros termoplásticos. Metin e colaboradores (METIN et al., 2004) observaram comportamento similar em compósitos $\mathrm{PP} /$ zeólita natural. Os autores investigaram o efeito da interação interfacial polímero-carga nas propriedades mecânicas dos compósitos, bem como a modificação da zeólita com silanos. A melhor adesão interfacial foi obtida com a utilização de agentes de acoplamento, cujos compósitos resultaram em melhores propriedades mecânicas.

Os valores máximos e mínimos obtidos nos experimentos para o alongamento na ruptura foram 24 e 7\%, para os compósitos contendo fibras não modificadas e fibras modificadas, respectivamente, sendo que para o PP, o valor obtido nas mesmas condições foi de $414 \%$. É comum observar decréscimo no alongamento na ruptura quando são incorporadas cargas em matrizes poliméricas, sendo este efeito mais pronunciado quando o polímero é passível de sofrer alongamento, como é o caso do PP. Vale ressaltar também que as cargas podem atuar como pontos de concentração de tensão, levando à fratura prematura do material.

O modelo de análise proposto pelo planejamento fatorial fracionário apresentou uma distribuição normal num intervalo de confiança de $95 \%$. A partir dos resultados obtidos, os melhores valores para as variáveis escolhidas estão apresentados na Tabela 2.

Tabela 2 - Níveis dos melhores valores das variáveis.

\begin{tabular}{lc}
\hline Variáveis & Níveis \\
\hline Composição da fibra & $15 \%$ \\
Modificação & Fibra modificada \\
Degasagem & Fechada \\
Fluxo de injeção & $3 \mathrm{~cm}^{3} \cdot \mathrm{s}^{-1}$ \\
Velocidade de ensaio & $50 \mathrm{~mm} \cdot \mathrm{s}^{-1}$ \\
\hline
\end{tabular}

Fonte: Dados do autor.

Para verificar o efeito da modificação superficial da fibra com o silano, a nível microscópico, a morfologia dos compósitos PP/FS foi avaliada por microscopia eletrônica de varredura. É válido ressaltar que estas fibras modificadas com viniltrimetoxissilano foram empregadas como reforço em matrizes elastoméricas de siliconas. Os espectros infravermelho confirmaram a presença 
dos grupos vinila na superfície das mesmas (SILVA; GONÇALVES; YOSHIDA, 2006). A Figura 3 ilustra as micrografias da superfície de fratura criogênica dos compósitos de PP reforçados com fibras não modificadas e modificadas com VTS.

Nos compósitos utilizando fibras não modificadas, (Fig. 3a), foram observadas regiões com lacunas na interface entre as fibras de sílica e a matriz de PP, o que é um indicativo de fraca adesão interfacial. Vale ressaltar, neste caso, que a interação entre as duas fases não é favorável, uma vez que as fibras são hidrofílicas, ou seja, apresentam grupos silanóis em sua superfície e, o PP, é constituído por cadeias apolares. Por outro lado, nos compósitos obtidos com as fibras modificadas com VTS, (Fig. 3b), foi constatado um melhor grau de molhabilidade e adesão entre as fases. Isto indica que a modificação da fibra com o VTS foi efetiva para promover a hidrofobização na sua superfície e, conseqüentemente, obter uma melhor interação com a matriz hidrofóbica de PP. A melhor adesão interfacial obtida entre PP e FS confirmou os maiores valores para o módulo de elasticidade nos compósitos contendo fibra modificada em relação a aqueles utilizando a fibra natural.

Figura 3 - Micrografias das superfícies de fratura de compósitos de PP reforçados com: (a) 15\% de fibras não modificadas e (b) $15 \%$ de fibras modificadas com viniltrimetoxissilano.

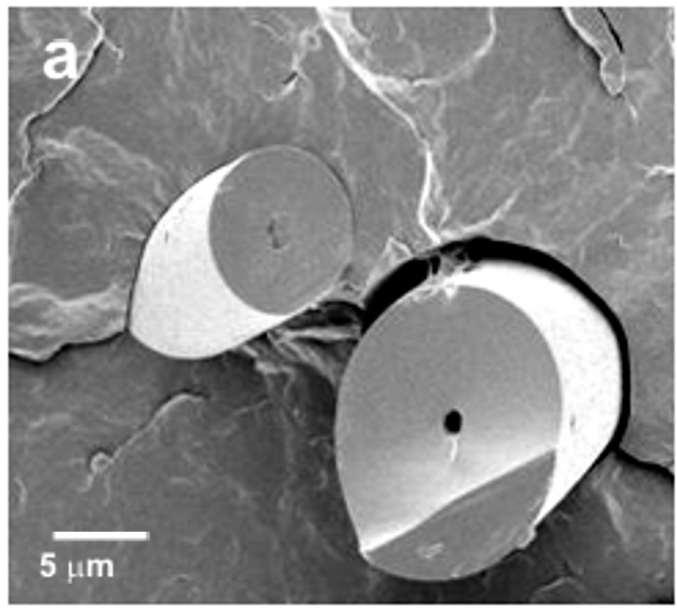

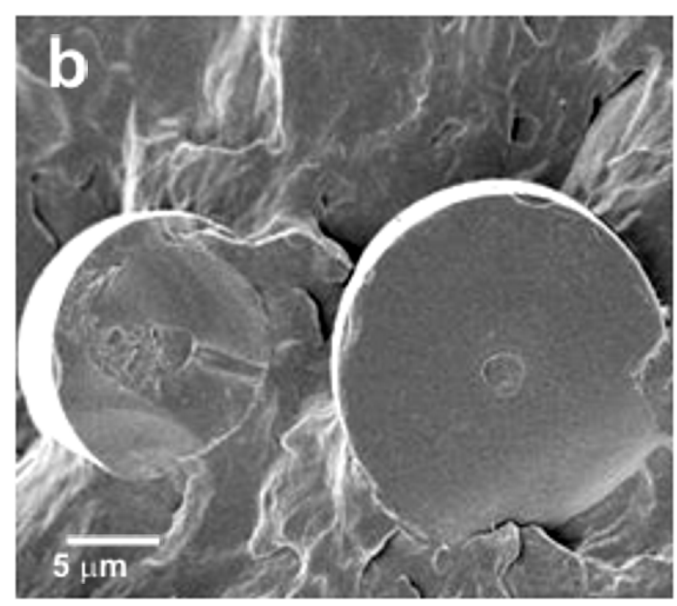

Fonte: Dados do autor.

\section{Conclusões}

Na obtenção de compósitos PP/FS, os resultados obtidos demonstraram que a interação na interface fibra-matriz é um aspecto importante na otimização das propriedades destes, e o VTS atuou como um agente de acoplamento eficiente neste sistema. O planejamento fatorial foi útil, uma vez que permitiu avaliar o efeito de algumas variáveis de composição e processamento nas propriedades mecânicas dos compósitos de polipropileno com fibras curtas de sílica.

\section{Agradecimentos}

Os autores agradecem à Braskem, Cerâmica São Caetano, Dow Corning do Brasil e Du Pont pelo fornecimento dos materiais, e ao CNPq pelo auxílio financeiro.

\section{Referências}

ARBELAIZ, A.; FERNÁNDEZ, B.; CANTERO, G.; LLANO-PONTE, R.; VALEA, A.; MONDRAGON, I. Mechanical properties of flax fibre/polypropylene composites. Influence of fibre/matrix modification and glass fibre hybridization. Composites: Part A: Applied Science and Manufacturing, Kidlinton, v. 36, p. $1637-$ 1644, 2005. 
American Society for Testing and Materials. "ASTM D-638: Standard Test Method for Tensile Properties of Plastics". West Conshohocken, 2003.

BALAS, F.; KAWASHITA, M.; NAKAMURA, T.; KOKUBO, T. Formation of bone-like apatite on organic polymers treated with a silane-coupling agent and a titania solution. Biomaterials, Surrey, v. 27, p. 1704-1710, 2006.

BARROS NETO, B.; SCARMINIO, I. S.; BRUNS, R. E. Como fazer experimentos: Pesquisa e desenvolvimento na ciência e na indústria. Campinas: Ed. UNICAMP, 2002.

BOUZA, R.; LASAGABASTER, A.; ABAD, M. J.; BARRAL, L. Effects of vinyltrimethoxy silane on thermal properties and dynamic mechanical properties of polypropylene-wood flour composites. Journal of Applied Polymer Science, New York, v. 109, p. 11971204, 2008.

CHAWLA, K. K. Composite-Materials-Science and Engineering. New York: Springer-Verlag, 1987.

HUFENBACH, W.; ADAM, F.; FISCHER, W. -J.; KUNADT, A.; WECK, D. Mechanical behaviour of textile-reinforced thermoplastics with integrated sensor network components. Materials \& Design, Surrey, v. 32, p. 4931-4935, 2011.

METIN, D.; TIHMINLIOGLU, F.; BALKÖSE, D.; ÜLKÜ, S. The effect of interfacial interactions on the mechanical properties of polypropylene/natural zeolite composites. Composites: Part A: Applied Science and Manufacturing, Kidlinton, v. 35, p. 23-32, 2004.

OTA, W. N.; AMICO, S. C.; SATYANARAYANA, K. G. Studies on the combined effect of injection temperatura and fiber content on the properties of polypropylene-glass fiber composites. Composites: Part A: Applied Science and Manufacturing, Kidlinton, v. 65, p. 873-881, 2005.

PLUEDDEMANN, E. P. Silane coupling agents. New York: Plenun Press, 1991.

REDONDO, S. U. A.; RADOVANOVIC, E.; GONÇALVES, M. C.; YOSHIDA, I. V. P. Eucalyptus kraft pulp fibers as an alternative reinforcement of silicone composites. I. Characterization and chemical modification of Eucalyptus fibers with organosilane coupling agent. Journal of Applied Polymer Science, New York, v. 85, p. 2573-2579, 2002.

ROHDE, M.; EBEL, A.; WOLFF-FABRIS, F.; ALTSTADT, V. Influence of Processing Parameters on the Fiber Length and Impact Properties of Injection Molded Long Glass Fiber Reinforced Polypropylene. International Polymer Processing, Munich, v. 26, p. $292-$ 303, 2011.
SENER, S.; ERDEMOGLU, M.; ASILTÜRK, M.; SAYILKAN, $\mathrm{H}$. The effect of silane modification on the adsorptive properties of natural pyrophyllite and synthetic titanium-based powders prepared by the sol-gel process. Turkish Journal of Chemistry, Ankara, v. 29, p. 487-495, 2005.

SEVER, K.; SARIKANAT, M.; SEKI, Y.; ERKAN, G.; ERDOGAN, U. H.; ERDEN, S. Surface treatments of jute fabric: The influence of surface characteristics on jute fabrics and mechanical properties of jute/polyester composites. Industrial Crops and Products, v. 35, p. 2230, 2012.

SILVA, V. P.; GONÇALVES, M. C.; YOSHIDA, I. V. P. Biogenic silica short fibers as alternative reinforcing fillers of silicone rubbers. Journal of Applied Polymer Science, New York, v. 101, p. 290-299, 2006.

THOMASON, J. L. The influence of fibre length and concentration on the properties of glass fibre reinforced polypropylene. 6 . The properties of injection moulded long fibre PP at high fibre content, Composites: Part A: Applied Science and Manufacturing, Kidlinton, v. 36, p. 995-1003, 2005.

UNAL, H.; MIMAROGLU, A. Influence of filler addition on the mechanical properties of nylon-6 polymer. Journal of Reinforced Plastics and Composites, Westport, v. 23, p. 461-469, 2004.

UNAL, H.; MIMAROGLU, A.; ALKAN, M. Mechanical properties and morphology of nylon-6 hybrid composites. Polymer International, London, v. 53, p. 56-60, 2004.

WANG, B.; LI, Q.; KANG, J. F.; PANG, J. F.; WANG, W.; ZHAI, J. P. Preparation and characterization of polypyrrole coating on fly ash cenospheres: role of the organosilane treatment. Journal of physics D-Appplied Physics, London, v. 44, p. 1-9, 2011 a.

WANG, J. C.; GENG, C. Z.; LUO, F.; LIU, Y. M.; WANG, K.; FU, Q. A., HE, B. B. Shear induced fiber orientation, fiber breakage and matrix molecular orientation in long glass fiber reinforced polypropylene composites. Materials Science and Engineering A-Structural Materials Properties Microstructure and Processing, Lausanne, v. 528, p. 3169-3176, 2011 b.

ZONG, Y.; YANG, X. G.; DAI, G. Design Simulation of Glass-Fiber-Loaded Flow in an Internally Spout-Fluidized Bed for Processing of Thermoplastic Composites. I. Flow Characterization. Industrial \& Engineering Chemistry Research, Washington, v. 50, p. 9181-9196, 2011.

Recebido em 24 Julho 2011-Received on July 24, 2011.

Aceito em 27 Dezembro, 2011 - Accepted on December 27, 2011. 